\title{
Multidimensional Model of Product Quality Formation
}

\author{
Julius Tchigirinsky ${ }^{1, *}$, Nadezhda Gribanova $^{1}$, Alina Gontar $^{1}$, and Nataly Chigirinskaya ${ }^{2}$ \\ ${ }^{1}$ Volgograd State Technical University, Technology of Machinery Department, 400005, Volgograd, \\ Russia \\ ${ }^{2}$ Volgograd State Technical University, High Mathematics Department, 400005 Volgograd, Russia
}

\begin{abstract}
Based on the analysis of the functional capabilities of modern information systems, the paper substantiates the applicability of multidimensional combined information structures for computer-assistant planning in machinery production. A multidimensional optimization model has been developed for elementary technological surface-treatment routing in the case of using complex quality indicators determined by the operational properties of products. The dimensions of its coordinate space are determined by the number of technical constraints that govern the quality of the product. The paper provides an example of an algorithm that uses logical algebra operations to reduce the coordinate space of the model and to reduce the computational complexity of the design task.
\end{abstract}

\section{Basic concepts}

The concept of quality in relation to mechanical engineering products includes "... a set of properties that determine their suitability to fulfill their functional purpose in a given range of changes in operating conditions" $[1,2,3,4]$. Simple and complex indicators are used to assess the quality of machines. In particular, mechanical engineering uses a system of simple indicators, which are subdivided into operational and production-and-technical ones $[1,2]$. In the group of production-and-technical indicators, there are indicators of dimensional accuracy, shape errors, geometric relationship, and the surface condition of machine parts.

Quality management is understood as "... a complex of influences on the process of creating products in order to ensure its quality by performing the following functions: - planning and quality control; - communication (information);- developing and implementing of measures; - making decisions on quality" [5]. Note that the concept of quality model is considered, in terms of the relevant regulatory document [5], as a description of a set of documents, the presence of which confirms the implementation of the management procedure, but not as a mathematical relationship between manufacturing conditions and production results.

In these definitions, we are interested specifically in the activities carried out at the initial stage of product creation - i. e., at the stage of production planning, in the process of

\footnotetext{
* Corresponding author: Julio-Tchigirinsky@yandex.ru
} 
technological routing. Regardless of the conditions of production planning - i. e., either arranging a new production line or modernizing an existing one - in the general chain of technological design measures, there is a particular stage $[3,4]$ of forming possible options for routing, comparing their technical and economic indicators, and choosing the optimal option [6].

It is known $[1,6]$ that the formation of quality indicators is influenced not only by the finishing stages of machining, but also by the entire sequence of technological operations. However, the issues of surface treatment sequence algorithmization with normalized quality requirements (hereinafter - a machining sequence, technological chain, elementary route) is not properly reflected by the methods of technological design.

\section{Routing based on probabilistic tables of accuracy}

We consider traditional flat tables of machining accuracy [2] herein as a baseline for constructing a machining sequence. Our studies [6, 7, 8 etc.] substantiate the statistical heterogeneity and hence the ambiguity of the data that make up the content of such accuracy tables. Insufficiency (insufficient completeness) of accuracy tables is determined by the lack of data on changes in the values of quality and accuracy criteria in the course of the technological process when executing each pair of adjacent machining methods. These two factors determine both the impossibility of using multidimensional data structures to build information tools for formalized routing and the impossibility of formalized routing itself.

The issue of insufficient homogeneity or, more strictly speaking, of insufficient statistical reliability of data on technological tolerances of individual machining methods is solved [6] by constructing accuracy tables adapted for a specific production. Clustering data, e.g., according to the technological capabilities of equipment, can significantly enhance the statistical reliability of the initial reference information, but, at the same time, significantly increases the volume of information arrays.

Formalization of individual routing can be carried out on the basis of approach [6], which provides for a quantitative assessment of changes in the normalized quality indicators in the course of the technological process from the workpiece to the surface of the finished product. The sequence of machining is formed from technological transitions that provide a consistent significant improvement in the quality of the treated surface during the technological process. Significant improvement is associated with the calculation of changes in the values of technological machining quality indicators and the statistical assessment of the reliability of such a change. The informational basis for machining sequence routing is made up of probabilistic tables of accuracy $[6,8]$.

The probabilistic table of accuracy is a three-dimensional information structure consisting of several flat layers (matrices, tables) of the same dimension $[6,8]$. The dimension (the number of rows and columns of the matrix) of the layer is determined by the number of different technological methods applicable to treating surfaces of a certain type. Each layer of the probabilistic table is a combination of the technological tolerance table of machining methods and the adjacency matrix of the oriented weighted complete graph. We consider the state of the surface formed after each performed technological transition to be the vertices of the graph, and technological transitions to be arcs. The weight characteristics of the arcs differ for different layers of the same probabilistic table, since they are determined by the values of the change in each separately considered machining quality indicator as a result of the corresponding $\left(j^{\text {th }}\right)$ technological transition after the $i^{\text {th }}$ one.

In the additional layers of the probabilistic table, statistical estimates of the significance of the change in the machining quality criterion can be indicated as the weights of the arcs the coefficient of variation of the criterion under consideration $V_{i j}(1)$ and the value of the 
probability of change in $\Phi\left(V_{i j}\right)$ calculated (2) from the value of the coefficient of variation in accordance with a so-called worst-case forecast [6].

$$
\Phi_{i j}=2 \cdot \min \left(\begin{array}{l}
\frac{1}{\sqrt{S_{i}^{2}+S_{j}^{2}}} \\
\frac{1}{\sqrt{2 \pi}} \cdot \int_{-\infty}^{\bar{X}_{j} \mid} V_{i j}^{-2} \cdot \exp \left(\frac{V_{i j}^{-2}}{2}\right) d V \\
0,5+\frac{1}{V_{i j} \cdot \sqrt{6}}+\frac{1}{12 \cdot V_{i j}^{-2}} \\
0,5+\frac{1}{2 \cdot V_{i j} \cdot \sqrt{3}}
\end{array}\right)-1,
$$

where $\bar{X}, S$ are the mean value and the standard deviation of the quality index respectively.

Indicators of quality and machining accuracy are considered as random $[6,7,8]$ values. Depending on the methods for analyzing the results of quality indicators measurements, we draw a distinction between discrete random values and continuous ones and accordingly a distinction among methods for determining quantitative indicators of changes [6] in the product quality in the course of the technological treatment.

Introducing two additional vertices - 'source' and 'target', - into the structure of a weighted directed graph, we pass to the concept of transportation network. Mathematical models and algorithms based on probabilistic tables of accuracy allow us to consider the process of elementary routing as an optimal transportation problem that requires linear programming methods for its formal solution. In fact, we propose to consider the process of multi-junction machining as a process of virtual displacement of a product from the state of the workpiece (the source of the transportation network) to the state of the finished product (target). As target functions, one can consider the following traditional optimization criteria: - machining productivity; - technological costs; - tool costs, etc., or, e. g., a probabilistic assessment of the reliability of a machining plan or the complexity of a technological chain.

A formalized approach to solving the problems of routing makes it possible to obtain reasonable forecasts regarding the quality and accuracy of machining, therefore, we are talking about managing the quality of products at the initial stages $[3,4]$ of technological preparation.

\section{Multidimensional model of product quality formation}

The probabilistic table of accuracy is a three-dimensional information array, in which, in the simplest case, the number of layers is equal to twice the number of normalized quality indicators - i.e., for each indicator, both a flat matrix of the values of the indicator change and a flat matrix of probabilistic estimates regarding the reliability of the change are formed. The algebraic model of the considered quality formation process is graphically represented in the form of a multidimensional transportation network, and the dimension of the coordinate space is determined by the number of normalized quality indicators.

E.g., for one normalized indicator, the transportation network built in the 'previous transition in the technological chain - next transition' coordinates is flat [6]. The coordinate space of the model is Cartesian since the machining quality indicators are considered as 
mutually independent random variables [8]. Each new index of machining quality introduced into the set of constraints increases the dimension of the coordinate space of the problem by adding a new axis to the existing coordinate system, which is orthogonal to all the others. The number of the nodes of the transportation network $\mathrm{N}$, equal to the number of technological transitions available for building a sequence, is associated with the maximum number of arcs by the known relation:

$$
N_{\partial y z}=N \cdot(N-1) / 2
$$

For a multidimensional directed graph, relation (3) changes as follows:

$$
N_{\partial y 2}=N^{k} \cdot\left(N^{k}-1\right) / 2
$$

The computational complexity of a search algorithm for forming a set of admissible 'source - target' routes is proportional to the value of factorial of $N_{\text {arc }}\left(N_{\text {arc }}\right.$ !).

E. g., for a set of $N=4$ technological transitions, the number of arcs of the multidimensional graph and the complexity of the search algorithm are related to the number of normalized quality indicators as follows:

\begin{tabular}{l|c|c|c|c} 
Number of quality indicators & 1 & 2 & 3 & 4 \\
\hline Number of intermediate network nodes & 3 & 15 & 63 & 255 \\
\hline Max. number of arcs & 6 & 120 & 2016 & 32640 \\
\hline Algorithm complexity estimate & 720 & $>10^{198}$ & $\infty$ & $\infty$
\end{tabular}

Obviously, traditional methods for formalizing the solution of the routing problem, applicable for one or two machining quality indicators, become inoperable with an increase in the number of normalized indicators.

Consider the possibility of reducing the computational complexity of the problem under study. The transformation scheme of the initial information array - i.e., the probabilistic table of accuracy - is shown in Fig. 1. In the traditional formulation, the problem of choosing a technological method with due regard to the probabilistic assessment of the possibility of obtaining the required machining quality is solved by the methods of the theory of fuzzy sets: the probabilistic assessment (2) is a so-called membership function. Using the concept of confidence probability, one can make a transition from the theory of fuzzy sets to the conventional theory of sets:

$$
\Phi_{i j}^{\prime}=\left\{\begin{array}{ll}
0, & \Phi_{i j}<\alpha_{\text {довер }} \\
1, & \Phi_{i j} \geq \alpha_{\text {довер }}
\end{array} .\right.
$$

The mathematical basis of the transformation is logical algebra. Let's replace, for each quality indicator, in the adjacency matrices of the machining graph, the value of the probabilistic estimate (arc weight) by a logical value: ' 0 ' as meant in 'machining is impossible' or ' 1 ' as meant in 'the result of machining is guaranteed'. Then term-by-term multiplication of the corresponding elements $\Phi_{i j}^{\prime}(5)$ from all the layers of the probabilistic table of accuracy will result in ' 1 ' in the meaning of 'the machining quality at the $j$-th transition is guaranteed for all normalized indicators' or ' 0 ' in all other cases. The adjacency matrix obtained as a result of such an operation defines a graph containing only arcs corresponding to technological transitions that guarantee the required machining quality. The number of arcs in the resulting network will be significantly reduced in comparison with the original structure, which will lead to a decrease in the complexity of the algorithm. 




step of transformation
the multidimensional model

1) in EACH layer, the calculated probabilities compared with the trust level and then the numerical values of the weights of the graph ares were replaced with logical ones:

if "less" - the weight of the arc is " 0 "; if "not less" - the weight of the arc is "1"



2) multiplied the corresponding elements of all layers and formed a "virtual" (resulting) adjacency matrix, in which was remained only "ALLOWED" vertices and ares was remained

3) in a resulting graph was found possible paths "source" $\rightarrow$ "target"

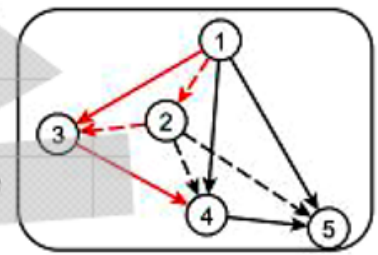

4) in the resulting adjacency matrix and in all layers of probabilistic table of accuracy, the all " 1 " were replaced with the corresponding values of arcs weight, in accordance with the selected optimization criterion, and the optimal solution was found

Fig. 1. Dimensional reduction scheme for a multidimensional mathematical model

The diagram (Fig. 1) demonstrates the following trends:

1) technological transitions "3 after 1" and "4 after 3" (elements of the adjacency matrices are highlighted in gray) ensure the fulfillment of only one quality indicator out of two normalized ones;

2) technological transitions "2 after 1 " and "3 after 2 " do not ensure the fulfillment of normalized quality indicators;

3 ) in the resulting adjacency matrix, transitions that do not ensure the fulfillment of a set of normalized quality indicators - "2 after 1", "3 after 1", "3 after 2", "4 after 3" (highlighted in red) - should be considered inadmissible; these transitions are not included in the machining sequence;

4) three-dimensional machining graph has been converted to a flat one, the number of intermediate vertices has been reduced by about five times, and the complexity of the task has been reduced.

\section{Conclusion}

The conventional approach to solving a technological design problem, which consists in constructing a multidimensional machining graph and finding possible paths in the graph, is practically applicable for few - no more than two,- normalized accuracy indicators. A significant reduction in the computational complexity of the route generation algorithm in a multidimensional graph becomes possible due to the construction of the resulting flat graph, including only those technological transitions, the execution of which ensures the 
achievement of the required accuracy normalized by all indicators without exception. The required machining quality is ensured with a probability of no less confidence.

\section{References}

1. Suslov, A. G. Design and technological support of quality and competitive ability of engineering products // Science Intensive Technologies in Mechanical Engineering. 2017. - No. 7 (73). - p. 25-28.

2. Technologist's Handbook/ ed. by A. G. Suslov - Moskow, Russia: Innovative Mechanical Engineering Publisher, 2019. - pp. 800.

3. Technological support for products development and production. Basic principles. State Standard R 50995.0.1-96 // Moskow, Russia: Standard Publishing, 2001.

4. Technological support for products development and production. Technological preparation of production. State Standard R 50995.3.1-96 // Moskow, Russia: Standard Publishing, 2001.

5. Quality systems. Model for quality assurance in design, development, production, installation and servicing. State Standard R ISO 9001-96 // Moskow, Russia: Standard Publishing, 1997.

6. Tchigirinsky, Ju. L. Technological route designing based with mathematical methods // Naukoemkie tekhnologii $\mathrm{v}$ mashinostroenii (High technologies in mechanical engineering). - 2018. - № 4 (82). - P. 13-20.

7. Firsov, I. V. CAD of routes the intermittent blade machining of chromium-containing steels and aluminum alloys // Problems of ensuring and improving the quality and competitiveness of engineering and aircraft engine products (TM-2015) : Proceedings of the VII Int. scientific and technical conf. (Bryansk, September, 21-23, 2015)/ Bryansk State Technical University [et al.]. - Bryansk, 2015. - P. 200-201.

8. Tchigirinsky, Ju. L., Chigirinskaya, N. V., Firsov, I. V. Structural optimization of technological route using simulation modeling// Proceedings of 2015 Int. Conf. on Mech. Eng., Automation and Control Systems (MEACS) (Tomsk, Russia, 14 Dec. 2015)/ Tomsk Polytechnic University. - P. 1-4. - DOI : 10.1109/MEACS.2015.7414925. 